\title{
Unmanned aerial vehicle with handover management fuzzy system for 5G networks: challenges and perspectives
}

\author{
Thalita Ayass ${ }^{1}$, Thiago Coqueiro², Tássio Carvalho² ${ }^{2}$ José Jailton ${ }^{2}$, Jasmine Araújo ${ }^{1}$, Renato Francês ${ }^{1}$ \\ 1 Institute of Technology, Federal University of Pará, Belém 66075-110, Brazil. \\ ${ }^{2}$ Computer Faculty, Federal University of Pará, Castanhal 68746-360, Brazil.
}

Correspondence to: Dr. Thalita Ayass, Institute of Technology, Federal University of Pará, Augusto Corrêa Street, 01, Guamá, Belém City, Pará State 66075-110, Brazil. E-mail: thalita_ayass@hotmail.com

How to cite this article: Ayass T, Coqueiro T, Carvalho T, Jailton J, Araújo J, Francês R. Unmanned aerial vehicle with handover management fuzzy system for $5 \mathrm{G}$ networks: challenges and perspectives. Intell Robot 2022;2(1):20-36.

https://dx.doi.org/10.20517/ir.2021.07

Received: 30 Aug 2021 First Decision: 22 Oct 2021 Revised: 8 Nov 2021 Accepted: 10 Feb 2022 Published: 22 Feb 2022

Academic Editors: Simon X. Yang, Jianjun Ni Copy Editor: Xi-Jun Chen Production Editor: Xi-Jun Chen

\begin{abstract}
The next generation of wireless networks, 5G, and beyond will bring more complexities and configuration issues to set the new wireless networks, besides requirements for important and new services. These new generations of wireless networks, to be implemented, are in extreme dependence on the adoption of artificial intelligence techniques. The integration of unmanned aerial vehicles (UAV) in wireless communication networks has opened several possibilities with increased flexibility and performance. Besides, they are considered as one of the most promising technologies to be used in the new wireless networks. Thus, UAVs are expected to be one of the most important applications to provide a new way of connectivity to the $5 \mathrm{G}$ network, and it is expected to grow from being a 19.3 billion USD industry in 2019 to 45.8 billion USD by 2025. In this paper, we provide a proposal of handover management on aerial $5 \mathrm{G}$ network utilizing the fuzzy system. The simulations performed prove the benefits of our proposal by QoS/QoE (quality of service/quality of experience) metrics.
\end{abstract}

Keywords: UAV, FANET, drone, fifth generation, fuzzy system, handover

\section{INTRODUCTION}

Unmanned aerial vehicles (UAVs) are considered as an interesting technology recently, mainly because of

The Author(s) 2022. Open Access This article is licensed under a Creative Commons Attribution 4.0

International License (https://creativecommons.org/licenses/by/4.0/), which permits unrestricted use, sharing adaptation, distribution and reproduction in any medium or format, for any purpose, even commercially, as long as you give appropriate credit to the original author(s) and the source, provide a link to the Creative Commons license, and indicate if changes were made. 
their deployment advantages and mobility ${ }^{[1]}$. There are two types of UAVs: fixed-wing and multi-rotor. The first is better applied to military applications, while the second is applied to provide wireless coverage to ground users. Moreover, UAVs are being applied in the following areas: efficient crop monitoring, delivery of goods, intelligent monitoring of places for security, carrying out surveys of various locations, developing a real-time map, coverage in telecommunications areas, and so ${ }^{2}{ }^{[2]}$. In addition, UAVs can support many Internet of Things (IoT) applications by providing real-time, accurate sensing/monitoring data ${ }^{[2]}$.

UAVs as aerial base stations will be an essential module for future wireless technologies, as they can support high data rate transmission for users located in disaster situations (e.g., after earthquakes, terrorist attacks, and so on) and when there is no typical cellular infrastructure.

Cellular networks are considered to be an alternative for drone communications because most commercial UAV systems employ IEEE 802.11 WLAN technology for sensor data, commands, and control, which operates in the unlicensed spectrum raising issues such as reliability and security ${ }^{[3]}$. Moreover, cellular networks with UAV-mounted base stations can enhance cellular networks, offering services where the traditional networks do not due to, e.g., costs. Besides all the applications cited above, UAVs as aerial base stations could be promptly dispatched, cheaply maintained, and easily maneuvered. UAVs can be used as end devices through cellular networks too ${ }^{[4]}$. Thus, UAVs could benefit the current network infrastructure, in terms of coverage, reliability, and security. There are also some ongoing standardization activities (security monitoring, rescue services, etc.) with $\mathrm{UAVs}^{[4]}$.

Some challenges to using UAVs as a main part of future mobile communications networks, serving as mobile users or mobile base stations, are interference, special mobility, and handover management. Unlike terrestrial networks, UAVs are mobile devices that move in a three-dimensional (3D) environment, which further complicates mobility issue ${ }^{[5]}$ as moving to a new location would disconnect the current users. Despite these problems, UAVs are becoming important for aerial communication ${ }^{[0,7]}$. Although UAVs offer numerous benefits for future wireless communication networks, their handover is a concern that must be studied deeply ${ }^{[5]}$.

The future generation wireless networks will be extremely dense and heterogeneous (with different technologies), likely equipped with moving and flying BSs (base stations). This makes the existing network planning techniques, which are mainly static and designed based on expensive field tests, not suitable for the future wireless networks. The utilization of artificial intelligence (AI) techniques for network planning has recently received interest in the research community. UAVs as aerial base stations for cellular networks are commonly used to support wireless coverage. However, an intelligent handover method must be proposed for UAV networks for when handover is triggered for a device moving to different UAVs.

One of the key premises in this development is the integration of AI into mobile communication networks. In this context, $\mathrm{AI}$ and machine learning techniques are expected to provide solutions for the various problems that have already been identified when UAVs are used for communication purposes such as channel modeling, resource management, positioning, interference from the terrestrial node, and handover.

This paper is structured as follows. Flying ad hoc network (FANET) concepts are discussed in Section 2. Section 3 outlines the FANET challenges and perspectives. The related works are presented in Section 4 . Section 5 describes the proposed handover management by the fuzzy system in detail and the results obtained from the simulation. Section 6 summarizes the conclusion and makes suggestions for future work. 


\section{FLYING AD HOC NETWORK}

Ad hoc networks, referred to by the IETF (Internet Engineering Task Force) as MANET (mobile ad hoc networks), have as their main characteristic the fact that they do not have infrastructure. As a result, all their functions must be performed by the devices. Thus, the devices that make up an ad hoc network must be able to communicate with each other acting as routers ${ }^{[1]}$.

Ad hoc networks are often used in scenarios where there is a need to quickly set up a network, usually where there is no proper infrastructure. The devices can move arbitrarily, unpredictably modifying the network topology, which requires a permanent adaptation and reconfiguration of routes so that the devices can still communicate with each other.

In the new context of fifth-generation networks $(5 \mathrm{G})$, a derivation of the ad hoc networks called FANET has emerged. FANETs are ad hoc networks composed of remotely controlled flying devices (UAVs) that communicate with each other ${ }^{[2]}$. Due to the flexibility, versatility, and even easy operation of FANETs, they are used for both military and civil applications, for example plantation control in agriculture, forest clearing, and city security (see Figure 1).

In recent years, because of technological advances in areas such as robotics, telecommunications, and computer networks, UAVs have emerged as alternatives in civil and military areas, providing several applications. Thus, UAVs are intended to improve or create a network infrastructure in places that are difficult to access, such as natural disaster areas or enemy territories. With this, FANET appears as an acceptable solution in this new context, allowing the collection of information in a flexible, fast, and reliable way.

One of the goals of FANETs is to create a cooperative network, using multiple UAVs to cover an area that cannot be covered by a single UAV. Thus, it is possible to create an aerial mesh network in which its devices (drones) communicate and transmit information with each other. Therefore, it is necessary to have reliable and stable communication between devices to maintain good levels of quality of service/quality of experience (QoS/QoE).

In FANETs, the mobility index is much higher than a traditional ad hoc network, leading to frequent topology changes. This is the reason FANETs must be self-configuring and self-organizing. Such a network must be prepared for sudden changes in its topology, organization, and even communication.

The mobility of UAVs and their spatial location are also very important for determining communication routes. With the motion, these routes are usually remade to continue with the interconnection of the UAVs. For this reason, routing must be done dynamically, and the routing protocol must be efficient and simple, increasing the autonomy of the UAVs and reducing the delay in data delivery between the drones.

UAVs are responsible for overflying the environment. They have sensors to collect information and can establish communication with each other more easily by finding fewer obstacles in their line of sight, reducing the number of UAVs needed to cover a certain area. However, weather conditions can impair communication due to wind, rain, and other factors.

FANETs have a high computational power. Thus, they have a greater capacity for transmitting information since in many cases they are responsible for transmitting information in real time (with videos from the monitored environment). Thus far, there are no specific routing protocols for FANETs. Traditional 


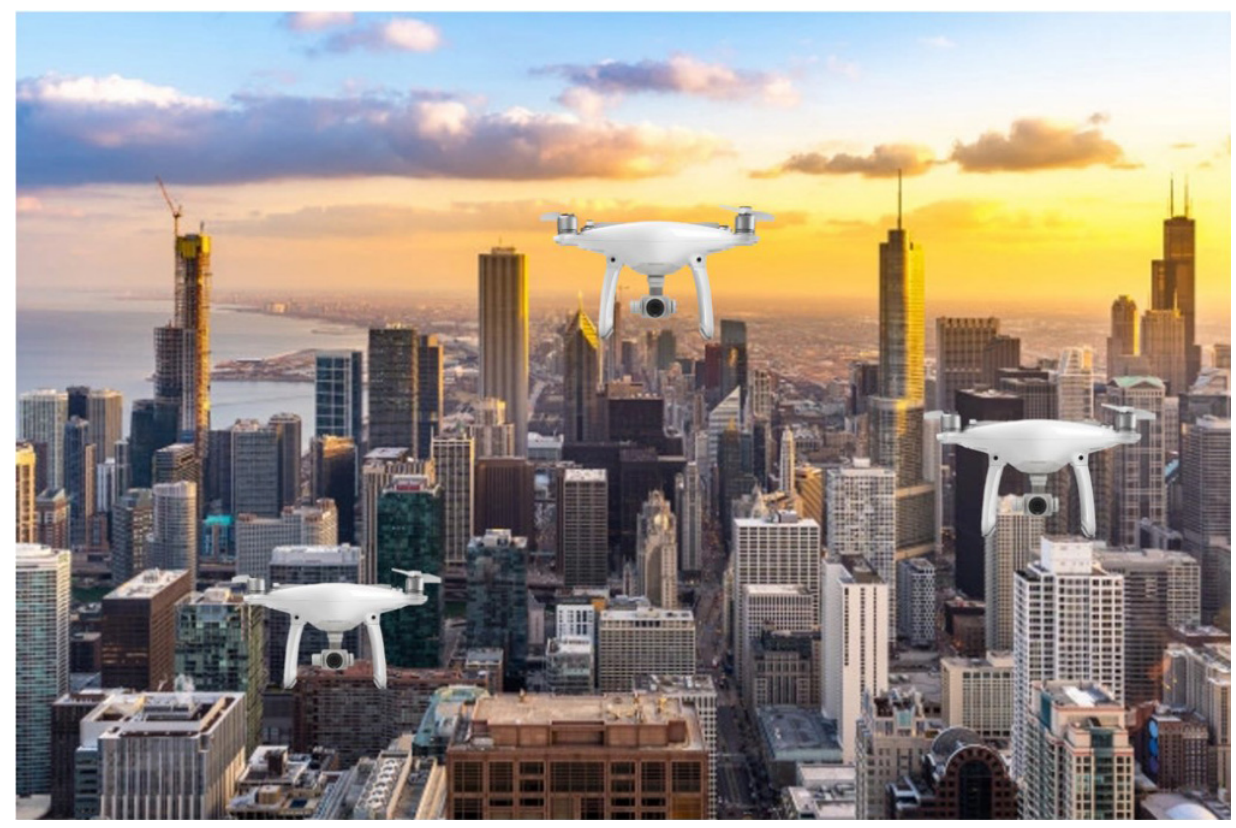

Figure 1. Flying ad hoc network scenario for city security monitoring.

protocols (AODV, OLSR, etc.) used in ad hoc networks are also used in this context [Figure 2].

Therefore, the location of devices within a coverage area directly impacts the performance of the network, which may improve or deteriorate according to their mobility. Thus, one of the main challenges to be solved in this type of network is the handover management after motion of UAVs.

Communication between UAVs depends a lot on their location since all information collected from the environment is concentrated in a relay node (which is responsible for relaying the data to a control center). Therefore, its positioning with respect to the other nodes is a strategic point to maintain a good performance of the network; it is not ideal that a UAV relay has excellent communication with some UAVs but poor communication with others in the network (see Figure 3).

Due to the high rate of mobility of UAVs in a FANET, updating the location of all nodes in the network is a critical factor. Network devices need to know the location of the other elements in real time; thus, in addition to the use of GPS (which on average sends the location once per second), UAVs have an inertial measurement unit, capable of sending its location in an interval smaller than the GPS at any time.

\section{CHALLENGES AND PERSPECTIVES}

The technology used in UAVs has great advantages for current generation telecommunications networks and provides a great framework of improvements, challenges, and promises for the next generations of wireless communications, especially in areas with difficult access or in regions lacking physical infrastructure, providing a structure and ensuring connectivity where terrestrial devices may fail. Many smart solutions are proposed in the literature, involving the context of using drones and UAVs. Some of these solutions promise implementations to adequately serve numerous services in addition to Internet data network communications and distribution, e.g., surveillance services, military systems, intelligent traffic control and distribution, and other important points including in the concept of smart cities. 


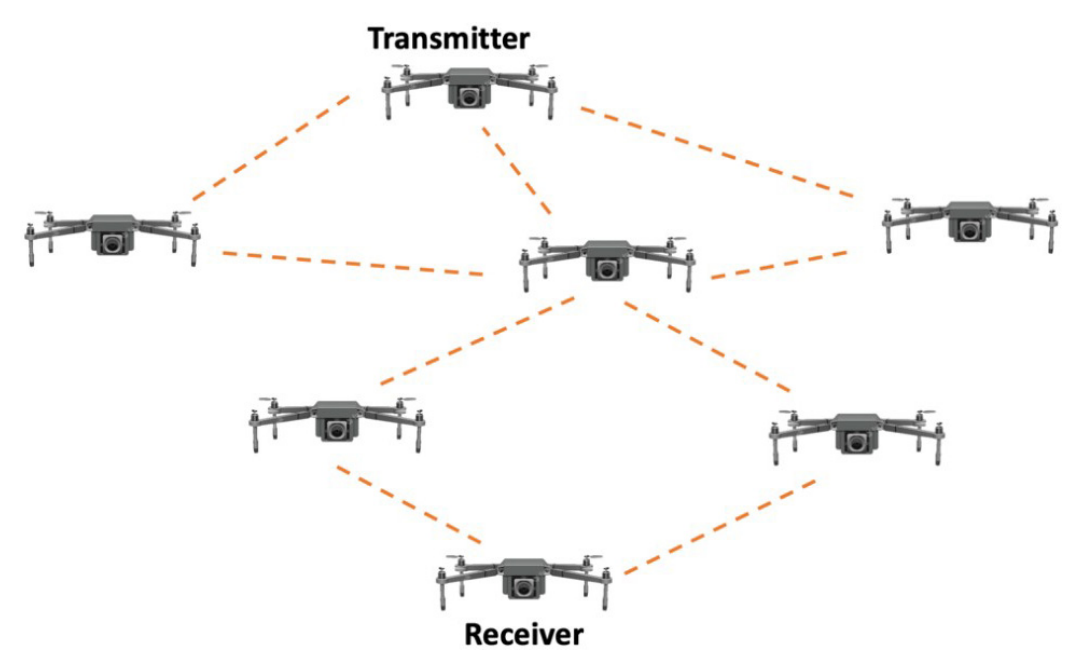

Figure 2. Flying ad hoc network routing protocol.

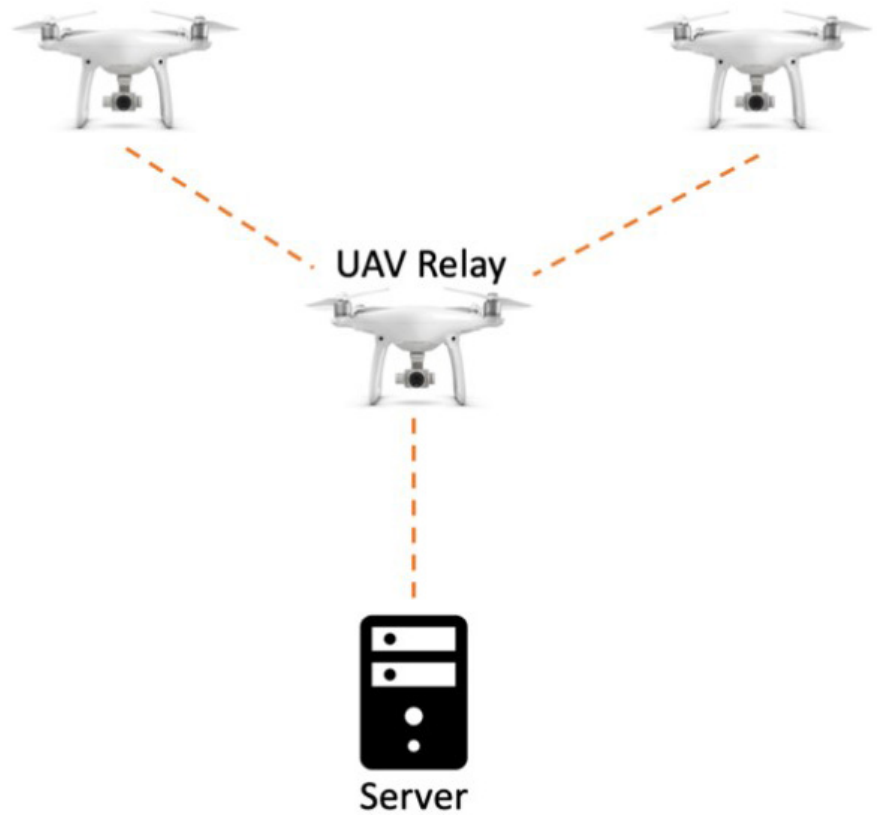

Figure 3. Unmanned aerial vehicle relay communication.

Although these systems have many advantages and benefits, they also present numerous challenges and perspectives, often due to inadequate or obsolete implementations, without updates or improvements.

\subsection{Challenges}

Communication networks through UAVs in the context of data communication and distribution present numerous challenges:

(1) Communication and transmission protocols are limited, due to the protocols currently used in communication networks and the Internet being obsolete for this new type of network and the way data are transmitted, with new characteristics. 
(2) There is an increased probability of errors and loss of information and data due to interference and signal strength problems, impairing communication, data packet delivery, and network system reliability.

(3) There are mobility management challenges, due to the high mobility of these devices, constant change of topology, and challenging coverage management and control processes. A sub-problem in this case is related to the increase in altitude, which can generate other challenges.

(4) The reliability of communication and handovers between devices and equipment, also due to the high mobility of UAVs, is lower, which can increase the delay, impair wireless communication, make it more difficult to maintain communication links with higher quality, and bring new problems in the heterogeneity of this type of networks, especially in the context of technologies linked to $4 \mathrm{G}$ and $5 \mathrm{G}$. UAVs experience dynamic channel swings and sudden changes due to high mobility and have constant problems with handover and ping-pong effects.

(5) Challenges regarding the battery capacity of the devices, their replacement, the transformation of the network and communications topology, and the computational and communications cost because of these constant changes, among others, also exist ${ }^{[8]}$.

\subsection{Perspectives}

However, in another direction, communications through UAVs also have numerous proposals and possibilities for the future, as is constantly observed in current academic works, providing new possibilities for multi-hop scenarios, which allow communication services for fixed and mobile devices and the creation of new scenarios and dynamic ranges, quickly and reliably.

Among the possibilities that go beyond a communication system, we can highlight: (1) the use of UAVs for people with special needs, providing visual information, among others, for those who need it; (2) delivery services, constantly speeding up the competitive system of delivery of letters and products or assisting in this type of need; (3) environmental monitoring systems, with sensors for agriculture, water resources, temperature, and other monitoring systems, providing intelligent and dynamic decision-making; (4) offering an important resource in military scenarios or places without infrastructure, including serving as a base in scenarios of DTNs (delay tolerant networks); and (5) intelligent transport systems, helping to monitor and control traffic, accidents, and other unexpected scenarios using UAVs. Other benefits include inspection of electrical systems, use in telepresence and telemedicine, assistance in disaster and accident scenarios, smart cities, etc.

Thus, it can be said that the use of UAVs in wireless networks is contributing and taking network communications to a new level, integrating existing $4 \mathrm{G}$ and $5 \mathrm{G}$ networks with mobile device systems that dynamically and constantly recreate new scenarios, providing topologies, greater ranges and transmission rates, airbase station services, supporting terrestrial communications networks, helping in communication between devices and IoT environments in healthcare systems, transport with the accident detection, communication between vehicles, and energy management $t^{[9]}$.

\section{RELATED WORK}

This section describes related published work on handover decision techniques on UAV networks. These are mainly about strategies to ensure an efficient handover to maintain service continuity and acceptable performance in delivering content to users. 
$\mathrm{Hu}$ et al. ${ }^{[10]}$ proposed an intelligent handover control method for UAVs in cellular networks. They introduced a deep learning model to predict the user's trajectory to provide mobility management. The handover decision is conceived by calculating the received signal power based on the predicted location and the characteristics of the air-ground channel, for accurate decision making. The simulation results demonstrate that the proposal's handover success rate was $8 \%$ higher than the traditional handover method.

Lee et al. ${ }^{[1]}$ emphasized that the traditional handover decision is not suitable for drones that move and communicate in $3 \mathrm{D}$ space. The drone's characteristics are considered as input parameters, namely the speed limit and coverage area, which are used as input in a fuzzy system for decision making on the handover. Thus, the calculation of the number of handover decisions showed that considering the parameters related to the terminal (drone) and the parameters related to the network has a positive effect on the handover decision.

Madelkhanova et al. ${ }^{[12]}$ developed a new algorithm based on Q-learning to manage the handover between airbase stations and static BSs, to maximize the total capacity of the UEs served by the air BSs. The Qlearning agent states are described in terms of the load of the ground bases and the reward function is defined in terms of the capacity of the UEs served by the air BSs. The results show an increase in the capacity of the UEs by up to $18 \%$ and $20 \%$ in the level of satisfaction with the solution. They also demonstrated that the Q-learning process converges quickly and only dozens of handovers are needed to achieve a significant gain.

Park et al. ${ }^{[13]}$ presented an efficient handover mechanism for aerial networks in three-dimensional space, which differs considerably from conventional two-dimensional schemes. The proposed scheme adjusts the height of a drone and the distance between drones. For this purpose, the probability of successful handover without interruption and the false probability of starting the handover were considered to evaluate the ideal coverage decision algorithm. The proposed method was the first attempt to offer a handover scheme for drones in three-dimensional space.

Bai et al.$^{[14]}$ pointed out that the support of drones in cellular networks has allowed a wide range of new applications for next-generation wireless systems. However, they discussed that these networks were traditionally designed to serve terrestrial users, which contributes to the emergence of challenges to support wireless communication by drones. As these devices experience increased interference and channel fluctuation, they must perform handover more frequently and are more susceptible to failure rate and pingpong during movement.

Faced with these challenges, the authors proposed an improved mobility management algorithm for drones, exploring pre-configured flight path information and their air channel properties. That is the proposal of a route-aware handover decision algorithm to minimize the failure and reduce the number of unnecessary handovers. The simulation results also demonstrate that the algorithm can reduce the ping-pong effect in certain cases.

Dong et al. ${ }^{[15]}$ proposed a scheme that dynamically adjusts the HO trigger parameters (handover) to reduce the number of unnecessary transfers. The scheme specifically considers the UAV sailing at a certain altitude and taking off. Experiments showed that the presented solution can significantly reduce the number of unnecessary HOs and improve network performance. They also showed that the channel quality between the UAV and the BS is very different from that on the ground, and therefore selecting the most appropriate target BS is also important. 
Goudarzi et al. ${ }^{[16]}$ stated that the main challenges of communications assisted by UAVs today are to have adequate accessibility in wireless networks through mobile devices with an acceptable quality of service based on user preferences. To this end, they presented a new method based on game theory to select the best UAV during the HO process and optimize the transfer between UAVs, decreasing end-to-end delay, transfer latency, and signaling overhead. The results demonstrate the effectiveness of the proposed approach in terms of handover numbers, cost, and delay.

Azari et al. ${ }^{[17]}$ recommended a machine learning-based approach for the HO mechanism and resource management for cellular-connected drones. They offered a machine learning-based solution that captures the correlations in temporal and spatial levels to help make HO decisions. Peng et al. ${ }^{[18]}$ proposed a solution based on machine learning for predicting node mobility. They used the classification of movements to different classes based on predicting the nodes near a future location.

In the work of Angjo et al. ${ }^{[5]}$, the handover decision is optimized gradually using Q-learning to provide efficient mobility and ping-pong support. The proposed scheme reduces the total number of handovers. Simulation results demonstrate that the proposed algorithm can effectively minimize the handover cost in a learning environment.

To avoid the ping-pong handover, Shakhatreh et al. ${ }^{[19]}$ proposed a weighted fuzzy self-optimization (WFSO) approach for the optimization of the handover control parameters. The HO decision relies on three considered attributes: signal-to-interference-plus-noise ratio, the traffic load of serving and target base station, and user equipment's velocity. The results indicate that the proposed WFSO approach significantly lowers the rates of HOPP, radio link failure, and HOF in comparison with the other algorithms found in the literature.

In this way, several studies have been conducted to address various types of $\mathrm{HO}$ issues, mainly in support of mobility management to reduce handover failures as well as to reduce the ping-pong effect. The ping-pong effect is the frequent connections and disconnections with the BS as the served device changes locations.

However, few proposals support energy efficiency. Battery capacity is one of the main limitations, becoming a critical factor for the continuity of the application. Therefore, effective power management is required for devices that operate on battery power. Some solutions such as wireless charging, solar charging technology, and even artificial intelligence techniques ${ }^{[9]}$ are indicated for effective energy management that provides longer missions.

Finally, Singh et al. ${ }^{[20]}$ proposed reinforcement learning (RL) based on an energy-aware ABS deployment algorithm. Dynamic movements of ABSs are managed by defining the user mobility pattern. However, this study does not support the quality of experience.

In this way, another critical factor would be the quality of user experience because it can measure the degree of quality of service through the user's perception. It is noteworthy that expectations about the satisfaction of different services and applications vary among different users. This means that QoE is an important attribute to be considered in the handover decision-making process.

Furthermore, research work carried out in recent years has focused on the field of artificial intelligence. Approaches based on machine learning and deep learning can ensure improvements in handover decision making and save computational costs ${ }^{[8]}$. On the other hand, handover decisions consider several parameters 
instantly, which advantages a fuzzy approach ${ }^{[8,11]}$.

Based on the survey of related literature (see Table 1), it is noted that the applicability of traditional handover schemes, as well as new propositions that support energy consumption and QoE, are still poorly investigated.

Thus, this paper proposes a fuzzy system strategy for handover decision, that is, combining support for mobility level, battery, displacement direction, throughput, and received signal strength indication (RSSI), proving that a fuzzy system is a promising technique for contributing to UAV networks.

\section{FANET FUZZY SYSTEM EVALUATION}

UAVs comprise a significant part of future wireless communication networks, acting as a mobile base station. While these devices provide several solutions related to mobile communication networks, UAVs also have numerous challenges, especially when it comes to handover management. Unlike terrestrial networks, drones are mobile devices that move in a $3 \mathrm{D}$ environment, which further complicates mobility issues.

Handover is one of the essential processes in wireless communication networks that guarantee continuous connection and quality of service while users are mobile. The criterion for the conventional handover decision is based primarily on the RSSI to indicate whether the device will remain attached to the current point of access or not. In the context of FANETs, this single premise for network selection can result in failures or even interruptions in service, since the UE can connect to a UAV with a low battery level, which is one of the most critical factors in these devices.

Similarly, high user mobility can compromise the quality of experience, due to the excessive number of handovers and the "ping-pong" effect that can direct the UE to a saturated network that offers low bandwidth.

Given this context, this work contributes with a study case that consists of presenting a system based on fuzzy logic as it is widely used in dynamic scenarios, as in the case of networks composed of UAVs, to assist in the decision making of handover in a FANET. The fuzzy system considers three input parameters: user speed, RSSI, and drones battery level. These inputs are processed by the inference system for the defuzzifier to evaluate and generate the decision-making outputs.

In fuzzy systems, the results are classified into a range from 0 to 1 . A value of 0 denotes an absolute exclusion, while a value of 1 denotes a complete correlation. The gap between the two extremes results in intermediate degrees of relevance. Elements can also belong to two or more defined sets, observing the values of the membership functions for each element.

One or more linguistic variables can be associated with the set of Fuzzy values, which represent the universe of the possibility of the expected results. In this work, the terms used to classify the outputs with the possibility of triggering the handover are: no, probably no, probably yes, and yes. The handover process is executed when the inference value is equal to or greater than 0.6 .

The system considers three input parameters that are processed by the inference system so that the defuzzifier can evaluate and generate the decision-making outputs. The first is related to the user's level of 
Table 1. Related works

\begin{tabular}{|c|c|c|c|c|c|}
\hline Paper & Proposed solution & $\begin{array}{l}\text { Ping-pong handover } \\
\text { reduction }\end{array}$ & $\begin{array}{l}\text { Energy efficiency } \\
\text { support }\end{array}$ & $\begin{array}{l}\text { Mobility management } \\
\text { support }\end{array}$ & $\begin{array}{l}\text { QoE } \\
\text { support }\end{array}$ \\
\hline [10] & Deep learning & No & No & Yes & No \\
\hline [11] & Fuzzy & No & No & Yes & No \\
\hline [12] & Q-learning algorithm & No & No & Yes & No \\
\hline [13] & $\begin{array}{l}\text { Coverage decision algorithm which controls } \\
\text { the coverage of each net-drone }\end{array}$ & No & No & Yes & No \\
\hline [14] & Route-aware handover algorithm & Yes & No & Yes & No \\
\hline [15] & Dynamic parameters to handover decision & No & No & Yes & No \\
\hline [16] & Cooperative game theory & Yes & No & Yes & No \\
\hline [17] & Machine learning-based solution & No & No & Yes & No \\
\hline [18] & Machine learning-based solution & No & No & Yes & No \\
\hline [5] & Q-learning based & Yes & No & Yes & No \\
\hline [19] & Fuzzy system & Yes & No & Yes & No \\
\hline$[20]$ & Reinforcement learning & No & Yes & Yes & No \\
\hline
\end{tabular}

QoE: Quality of experience.

mobility and indicates how long a mobile device remains in the coverage area of a station. The faster the device travels, the less time it will be connected to that access point. This first input is divided into three sets of linguistic values: slow (range 0-1.5 m/s), moderate (1.3-3 m/s), and fast (2.5-4 m/s).

The second input refers to the received signal level, represented by RSSI. This is a factor used to assess how likely the device is to disconnect from the access point if the signal strength is weak. In this metric, signal levels are defined for language sets as follows: weak (-120 to $-100 \mathrm{dBi})$, moderate $(-115$ to $-65 \mathrm{dBi})$, and strong (>-72 dBi).

The last input metric considers the drone's flight range, which is linked to how long the devices can remain in operation. This is an important criterion because, given the knowledge of the remaining time each UAV can still operate, unnecessary transfers are avoided for those drones that are in the unloading phase and will not be able to continue the service. For this parameter, the defined sets are: low (0-10 min), medium (8-20 $\mathrm{min})$, and high (18-30 min) battery levels.

Given the inputs, the fuzzy inference system will determine the outputs according to the set of 27 rules previously established from the combination of the three parameters. In this work, the Gaussian membership function is applied to all inputs and outputs. This function is chosen because of its characteristic of reducing the noise of input variables and its ability to represent real-world phenomena more naturally.

The output of the fuzzy system indicates the probability of the mobile device starting the handover process. In general, if a user has high mobility and high levels of RSSI, the transfer process to another network will not occur. The system indicates a trend of execution of the handover, as its inference value is equal to 0.6.

In the 3D surface graphics in Figure 4, it is possible to visualize the relationship between the chosen parameters. The region in blue corresponds to a user with high mobility and excellent signal strength. In this context, the handover process will not trigger. The yellow region indicates the opposite, the user with low speed and receiving a bad signal; in this case, the handover is executed. 


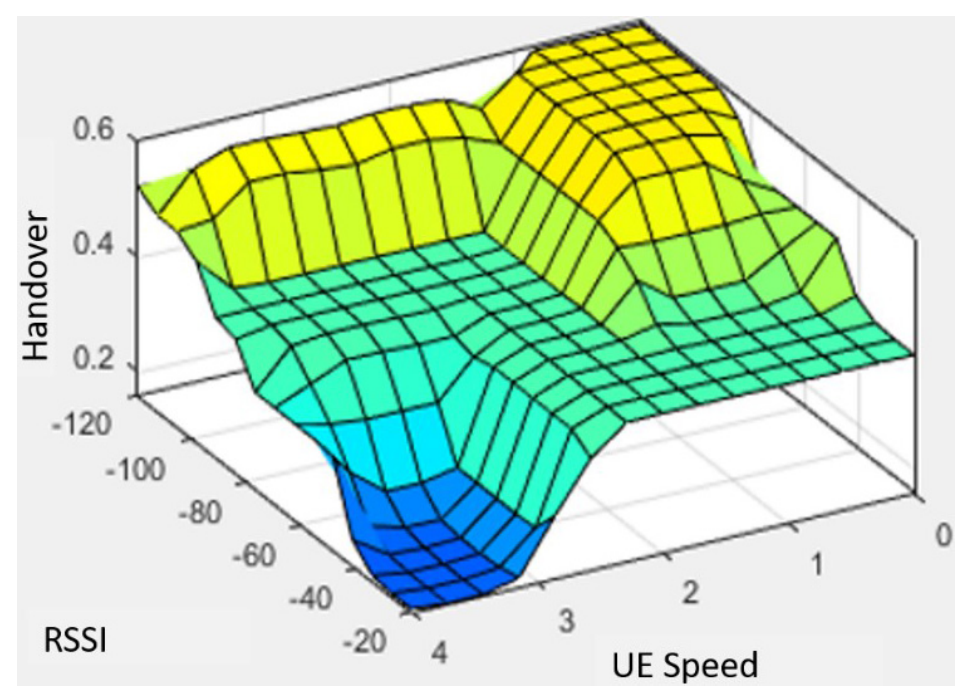

Figure 4. Inference fuzzy system.

The fuzzy system was implemented using the Matlab Fuzzy logic toolbox, where its inputs were defined and, after going through the defuzzification process, produced the numerical outputs that indicated the tendency to carry out the handover or not. To evaluate the performance of the network, according to the outputs that were indicated by the fuzzy system as being ideal for the handover decision, the technique used was to implement the scenarios in the simulation environment of the Network Simulator 2 (NS2) tool. The UAVs were placed at the same height of $100 \mathrm{~m}$, in an area of $1000 \mathrm{~m} \times 1000 \mathrm{~m}$, as shown in Figure 5 . In the simulation, a WI-FI network is considered where the UAVs serve as access points to promote the connection of users within a given environment, according to the displacement of the UEs. The main parameters used in simulation are summarized in Table 2.

To better understand the results, the evaluation considered the network throughput metric to verify the behavior of the proposal through the solution that was based on fuzzy logic for handover decision making.

In a first scenario, CBR-type applications were received by mobile users through the WI-FI interface enabled by UAVs that are operating as a network access point. The scenario was simulated by comparing the traditional handover process, which prioritizes RSSI as a transfer trigger, and handover from the proposed fuzzy architecture.

It was considered a high mobility environment within the UAVs' coverage area. In this context, it can be seen from the graph in Figure 6 that, by the traditional handover method, the UEs were subject to the pingpong handover effect and suffered a lot of instability in the connection. This behavior is perceived by the fact that the conventional handover model does not consider parameters that are characteristic of FANETs, especially regarding the UAV battery.

It is noticed that, between Seconds 90 and 120 of the simulation, there was an interruption in the service, caused by the unloading of the UAV. Even though the UE is reconnected from Second 120, right after the device suffers another disconnection because, even with good signal strength, the UAV was in full unloading phase. Differently, the handover proposed using the fuzzy system parameters that meet the characteristic requirements of UAVs, such as battery time, proved to be efficient when selecting a new network. 
Table 2. Simulation parameters

\begin{tabular}{ll}
\hline Parameter & Value \\
\hline UAV & 12 \\
Access technology & IEEE 802.11 g \\
Propagation model & Shadowing \\
Pathloss & $2(\mathrm{~dB})$ \\
Shadowing deviation & $4(\mathrm{~dB})$ \\
Mobility model & Random waypoint \\
Application & CBR/Video \\
Rate & $54 \mathrm{Mbps}$ \\
Simulation time & $130 \mathrm{~s}$ \\
\hline
\end{tabular}

UAV: Unmanned aerial vehicle.

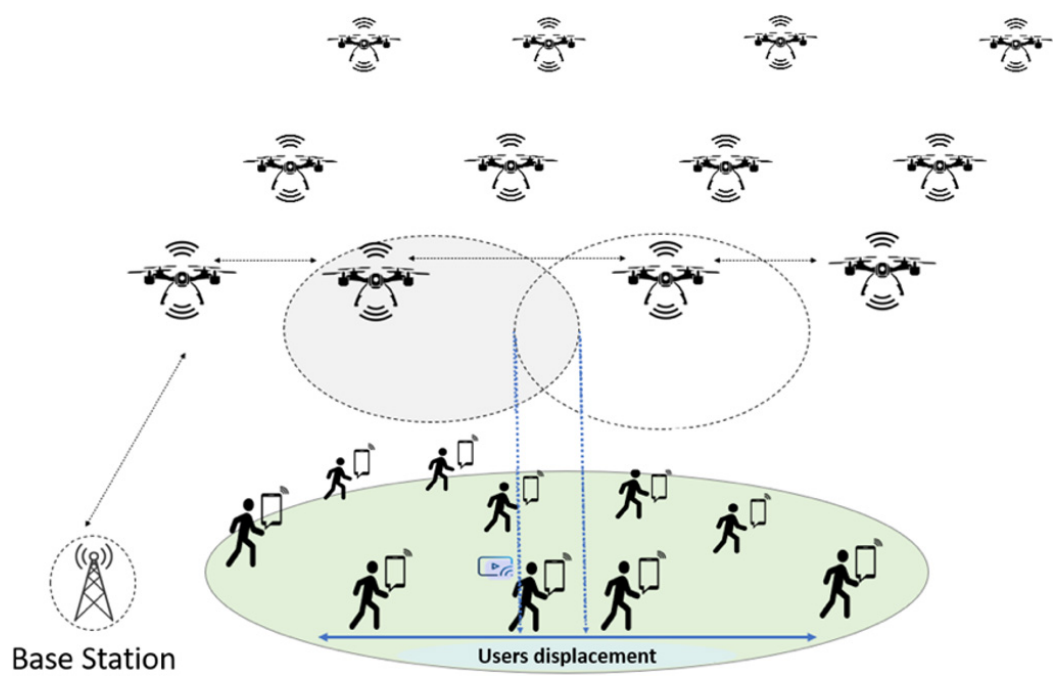

Figure 5. Flying ad hoc network scenario simulation.

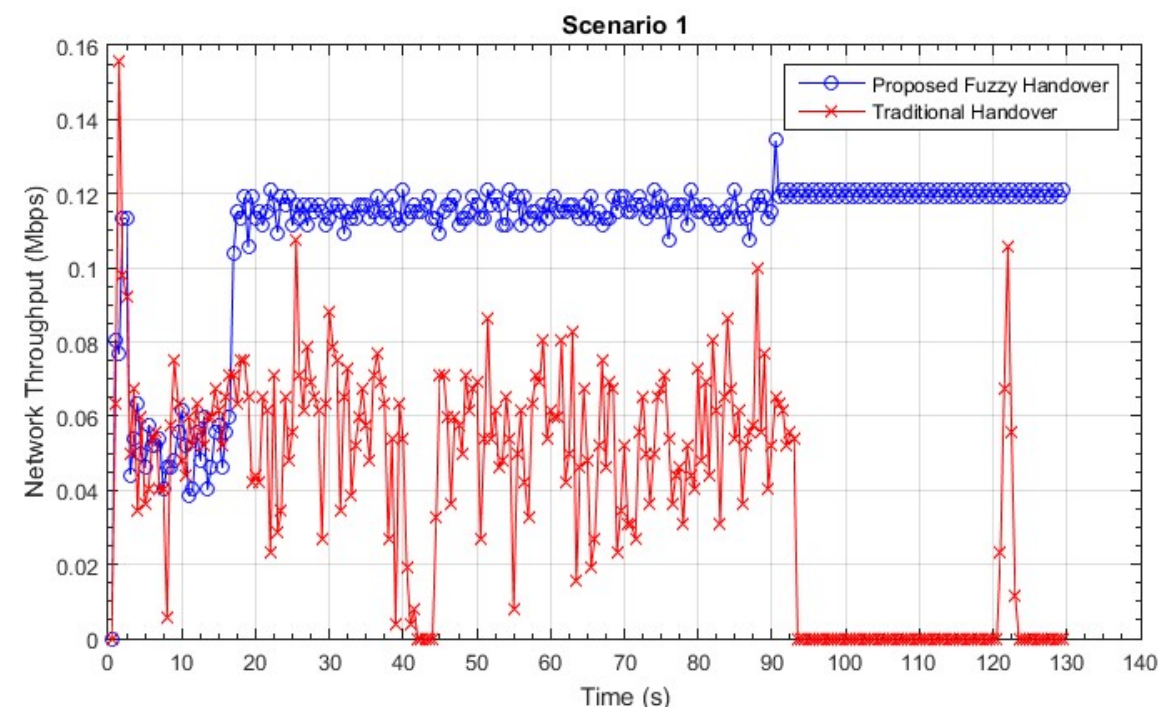

Figure 6. Throughput of Network I. 
From the criteria established in the architecture, it is noted that at around Second 19 of simulation the handover is performed, causing the average flow rate to reach twice the value of the previous connection and remain stable until the end of the simulation. This better performance is possible due to the management made by the proposal of handover with the fuzzy system, which prevents the UE from selecting a new access point where the UAV is about to discharge, even if it presents good signal strength.

In the second scenario illustrated in Figure 7, the network was subjected to a greater demand for data due to the increase in the number of users overloading the network. It is possible to see that, without the proposed solution, the network presented an even worse performance than in the previous scenario, where the flow rate drops drastically when using the traditional handover model.

The running application is also CBR type, and, by the traditional model, the transfer was made to the nearest network, even though there was no interruption in the connection. The new network was more overloaded and ended up causing the throughput to be below $0.1 \mathrm{Mbps}$. Conversely, the proposed method performed the handover only when necessary and maintained a stable connection when selecting a better network.

As in the first scenario, the handover with intelligent management of parameters by the fuzzy system was more efficient as it managed to maintain a constant connection, in addition to identifying the best access point and promoting a better flow rate to the UE from Second 90 of the simulation.

The study also analyzed the effects of the traditional process of handover and the one proposed by fuzzy inference, through simulations involving video application. To evaluate the quality of the media received, the QoE results in the same previous scenarios were compared. The video used in the simulation has a resolution of $176 \times 144,1000$ frames, and decoding in YUV 4:2:0 format, which stands for the color difference encoding system whether composite or component.

In this way, the peak signal-to-noise ratio (PSNR), structural similarity metric (SSIM), and video quality metric (VQM) metrics were considered, being these objective metrics that complement each other, to assess the impacts of signal degradation in the original video with the reference when the traditional handover was performed, as well as the proposed one. At the end of the transmission, the values of the metrics in question were calculated and displayed frame by frame.

PSNR has a range of values between 0 and $100 \mathrm{~dB}$. For values above $30 \mathrm{~dB}$, it is understood that the video has good quality. On the other hand, videos that are below the $20 \mathrm{~dB}$ range are considered of poor quality. For the network in the first scenario, Figure 8 shows the PSNR values for each frame of the video. Comparison with the original file shows that the PSNR of the video received had an average of $21.3 \mathrm{~dB}$ in the traditional handover, classifying it as a low-quality video. Differently, the PSNR for the video with the Fuzzy criteria obtained an average of $42.41 \mathrm{~dB}$, characterizing it as being of excellent quality.

The range of SSIM values is between 0 and 1, where 1 represents the exact correlation with the original image. Figure 9 shows the SSIM values of frames in the traditional handover obtaining an average of 0.59. For the proposed handover, the result obtained was 0.98 , being very close to 1 , which is the accepted value for a perfect correlation of images. In this sense, it could be seen that the reference video had low distortion for this parameter. 


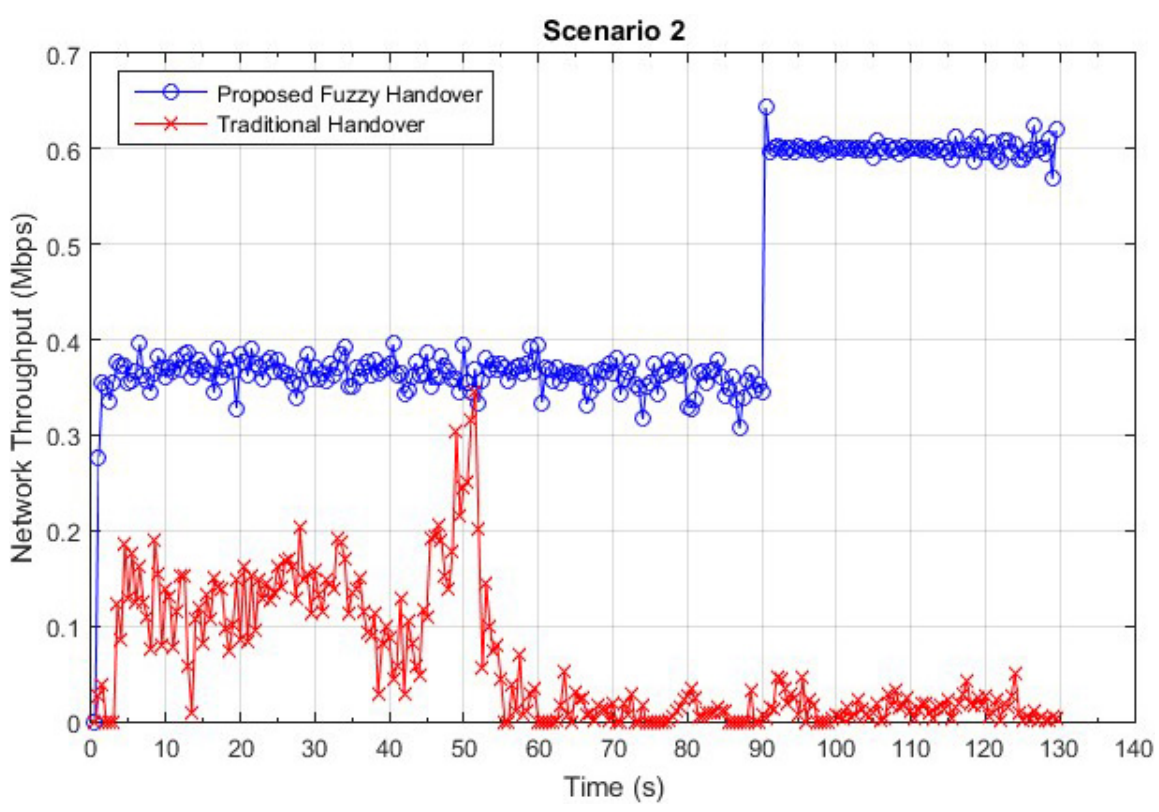

Figure 7. Throughput of Network II.

A

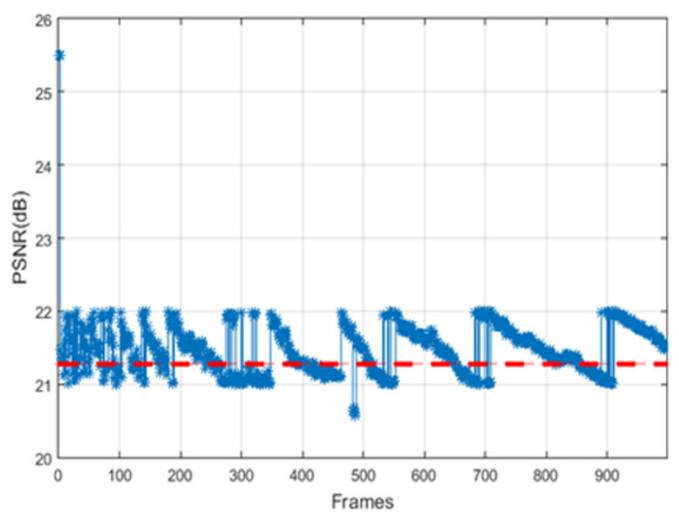

B

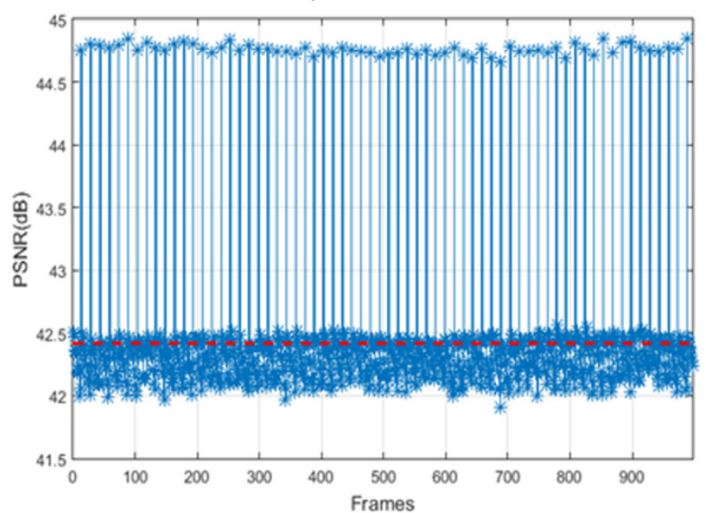

Figure 8. Peak signal-to-noise ratio traditional (A) and fuzzy handover (B).

The evaluation of the video quality of VQM is illustrated in Figure 10. For this metric, o characterizes the best possible value. The result obtained after the evaluation shows that the average was equal to 6.01 for the handover without the fuzzy system. The proposal achieved an average VQM of 0.50, which indicates that the video did not suffer considerable degradation in this sense, being close to the ideal value of 0 .

The superiority in the quality of the transmitted video that considers the HO by the fuzzy system can also be seen visually, making a frame-by-frame comparison between the original and received video in the two methods covered in the work. In Figure 11, Frame 305 presents degradations and distortions in the pixels of the video transmitted over the network without supporting the efficient handover scheme. However, the same frames remained undistorted in the video that was broadcast considering the fuzzy proposal. 

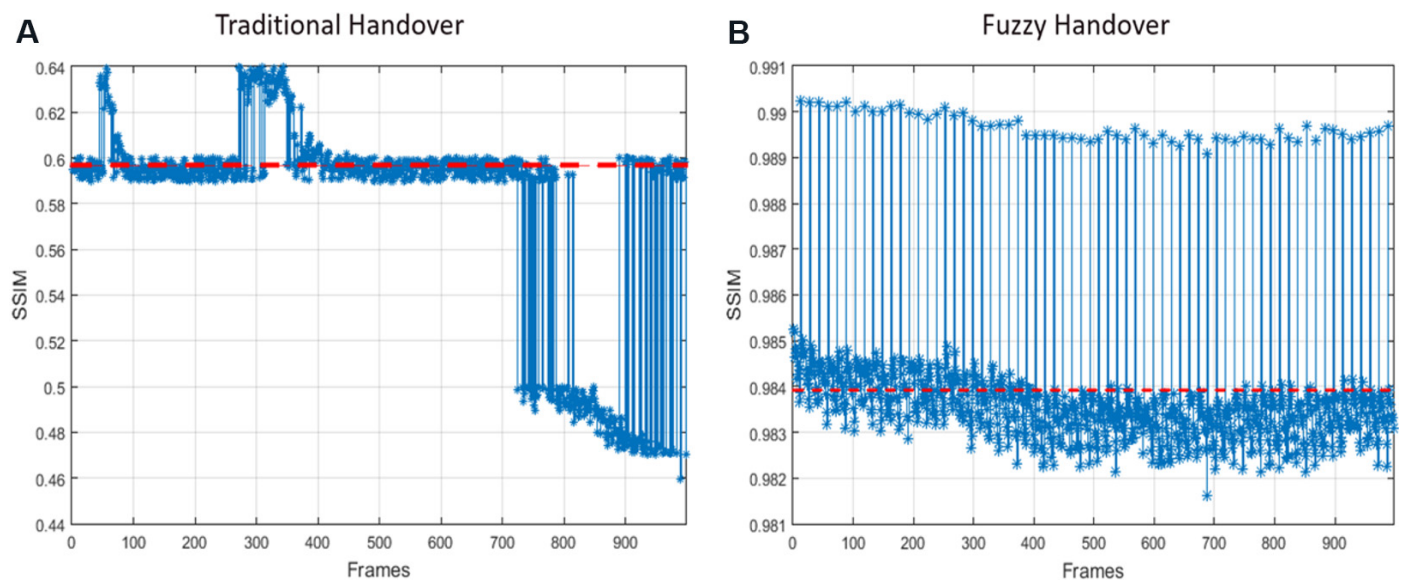

Figure 9. Structural similarity metric traditional (A) and fuzzy handover (B).
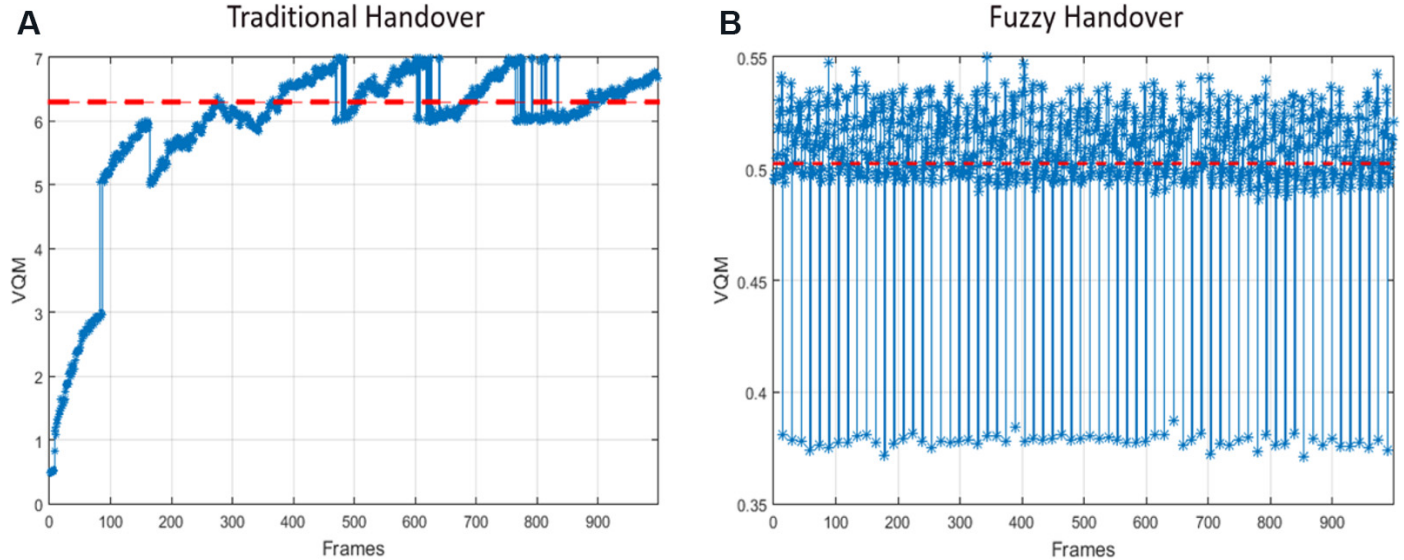

Figure 10. Video quality metric traditional (A) and fuzzy handover (B).
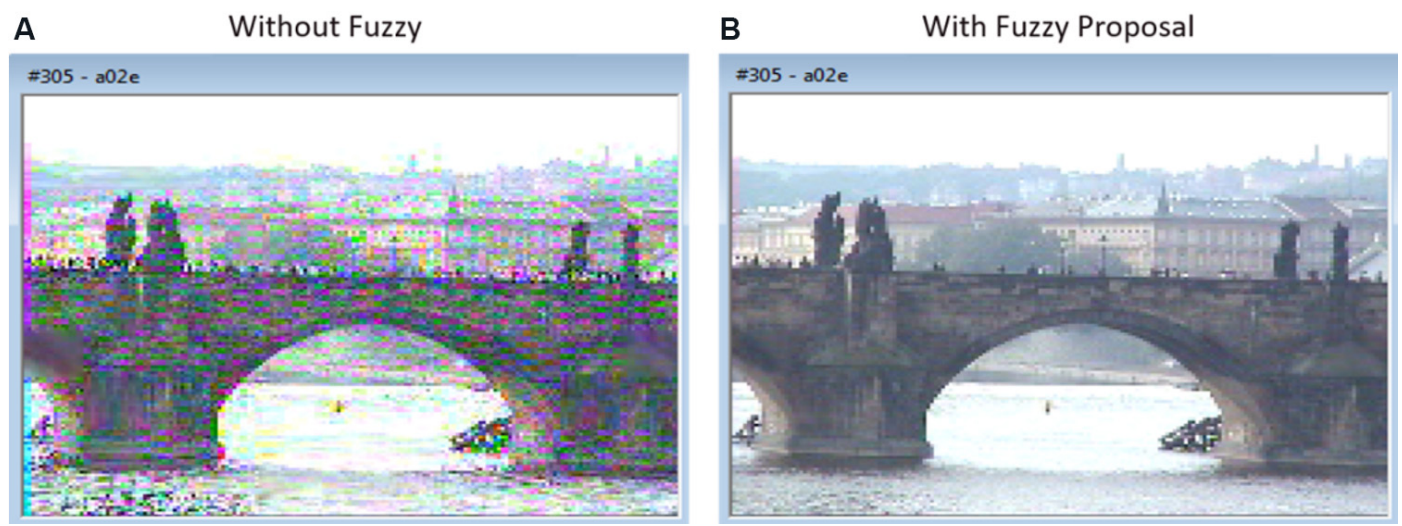

Figure 11. Frames comparison without fuzzy (A) and with the fuzzy proposal (B).

From the simulations and evaluative analysis on the performance of both the conventional handover model and the proposed one, it is possible to see the traditional model does not satisfy the peculiarities of these networks and their components, causing the low quality of service, compromising the quality of experience of the user, and not guaranteeing a transparent handover, which impacts the continuity of the service. 
By the proposed architecture, the handover process proved to be effective to mitigate handover issues in FANETs, since it achieved superior results in both QoS and QoE parameters, proving itself as a viable proposal.

\section{CONCLUSIONS}

In the context of fifth-generation networks, new connectivity alternatives emerge, such as the use of UAVs as an access point, mainly for locations with difficult access or without available network infrastructure.

To set up fast and temporary networks, FANETs are used in different scenarios to provide $5 \mathrm{G}$ access to users, but, due to the mobility of UAVs and the users themselves, the network topology is constantly modified. The constant topology changes in FANET due to mobility can disrupt the user's connection.

Thus, this paper proposes a FANET as an alternative way of connecting to $5 \mathrm{G}$ networks, in which drones work as access points for users. The paper also proposes the use of a fuzzy system for UAV mobility management for anticipating handovers to avoid network connection breaks.

The results prove a better performance when compared to a traditional FANET (without the use of the fuzzy system). The results were proven through the throughput metric and QoE metrics (for video transmissions), as well as the shown frames of transmitted videos. As future work, we intend to use other artificial intelligence techniques, as well as other wireless transmission technologies.

\section{DECLARATIONS}

\section{Authors' contributions}

Literature review, research/methodology design, writing the manuscript from initial writing until submission, revision of the manuscript according to reviewers' comments, data collection, result analysis and interpretation: Ayass T

Literature review, writing the manuscript from initial writing until submission, revision of the manuscript according to reviewers' comments, result analysis and interpretation: Coqueiro $\mathrm{T}$

Formulation of the initial research proposal, research/methodology design, writing the manuscript from initial writing until submission, revision of the manuscript according to reviewers' comments, result analysis and interpretation: Carvalho $\mathrm{T}$

Formulation of the initial research proposal, research/methodology design, technical guidance and assistance to methodology, writing the manuscript from initial writing until submission, revision of the manuscript according to reviewers' comments, co-researcher of the student: Jailton J

Formulation of the initial research proposal, literature review, technical guidance and assistance to methodology, writing the manuscript from initial writing until submission, co-researcher of the student: Araújo J

Formulation of the initial research proposal, technical guidance and assistance to methodology, researcher/supervisor of the project and student: Francês R

\section{Availability of data and materials}

Not applicable.

\section{Financial support and sponsorship}

None. 


\section{Conflicts of interest}

All authors declared that there are no conflicts of interest.

\section{Ethical approval and consent to participate}

Not applicable.

\section{Consent for publication}

Not applicable.

\section{Copyright}

(c) The Author(s) 2022.

\section{REFERENCES}

1. Carvalho T, Jailton Júnior J, Francês R. A new cross-layer routing with energy awareness in hybrid mobile ad hoc networks: a fuzzybased mechanism. Simul Model Pract Theory 2016;63:1-22. DOI

2. Noorwali A, Awais Javed M, Zubair Khan M. Efficient UAV communications: recent trends and challenges. Comput Mater Contin 2021;67:463-76. DOI

3. Fakhreddine A, Bettstetter C, Hayat S, Muzaffar R, Emini D. Handover challenges for cellular-connected drones. DroNet'19: Proceedings of the 5th Workshop on Micro Aerial Vehicle Networks, Systems, and Applications; New York: Association for Computing Machinery. 2019. p. 9-14. DOI

4. Enhanced L T E support for a e ria 1 vehicles. A v a lable from : https://portal.3gpp.org/desktopmodules/Specifications/SpecificationDetails.aspx?specificationId=3231 [Last accessed on 17 Feb 2022].

5. Angjo J, Shayea I, Ergen M, Mohamad H, Alhammadi A, Daradkeh YI. Handover management of drones in future mobile networks: $6 \mathrm{G}$ technologies. IEEE Access 2021;9:12803-23. DOI

6. Zeng Y, Guvenc I, Zhang R, Geraci G, Matolak DW, UAV communications for 5G and beyond. Hoboken: Wiley-IEEE Press; 2020. DOI

7. Saad W, Bennis M, Mozaffari M, Lin X. Wireless communications and networking for unmanned aerial vehicles. Cambridge: Cambridge University Press; 2020. DOI

8. Li B, Fei Z, Zhang Y, Guizani M. Secure UAV communication networks over 5G. IEEE Wireless Commun 2019;26:114-20. DOI

9. Sharma A, Vanjani P, Paliwal N, et al. Communication and networking technologies for UAVs: a survey. J Netw Comput Appl 2020;168:102739. DOI

10. $\mathrm{Hu} \mathrm{B}$, Yang H, Wang L, Chen S. A trajectory prediction based intelligent handover control method in UAV cellular networks. China Communications 2019;16:1-14. DOI

11. Lee E, Choi C, Kim P. Intelligent handover scheme for drone using fuzzy inference systems. IEEE Access 2017;5:13712-9. DOI

12. Madelkhanova A, Becvar Z. Optimization of cell individual offset for handover of flying base station. 2021 IEEE 93 rd Vehicular Technology Conference (VTC2021-Spring); 2021 Apr 25-28; Helsinki, Finland. IEEE; 2021. p. 1-7. DOI

13. Park K, Kang J, Cho B, Park K, Kim H. Handover management of net-drones for future internet platforms. Int J Distrib Sens Netw 2016;12:5760245. DOI

14. Bai J, Yeh SP, Xue F, Talwar S. Route-aware handover enhancement for drones in cellular networks. 2019 IEEE Global Communications Conference (GLOBECOM); 2019 Dec 9-13; Waikoloa, HI, USA. IEEE; 2019. p. 1-6. DOI

15. Dong W, Mao X, Hou R, Lv X; Li H. An enhanced handover scheme for cellular-connected UAVs. 2020 IEEE/CIC International Conference on Communications in China (ICCC); 2020 Aug 9-11; Chongqing, China. IEEE; 2020. p. 418-23. DOI

16. Goudarzi S, Anisi MH, Ciuonzo D, Soleymani SA, Pescape A. Employing unmanned aerial vehicles for improving handoff using cooperative game theory. IEEE Trans Aerosp Electron Syst 2021;57:776-94. DOI

17. Azari A, Ghavimi F, Ozger M, Jantti R, Cavdar C. Machine learning assisted handover and resource management for cellular connected drones. Available from: http://arxiv.org/abs/2001.07937 [Last accessed on 17 Feb 2022].

18. Peng H, Razi A, Afghah F, Ashdown J. A unified framework for joint mobility prediction and object profiling of drones in UAV networks. J Commun Netw 2018;20:434-42. DOI

19. Shakhatreh H, Sawalmeh AH, Al-fuqaha A, et al. Unmanned aerial vehicles (UAVs): a survey on civil applications and key research challenges. IEEE Access 2019;7:48572-634. DOI

20. Singh S, Sandhu MK. A review over existing handover decision systems for drones in wireless network. Available from: http://www.ijstr.org/final-print/mar2020/A-Review-Over-Existing-Handover-Decision-Systems-For-Drones-In-Wireless-Network.pdf [Last accessed on 17 Feb 2022]. 